\title{
Joint Power Allocation for Coherent Downlink Coordinated Transmission
}

\author{
Shiyuan $\mathrm{LI}^{1}$, Qimei CUI ${ }^{1}$, Harald Haas ${ }^{2}$, Xiaofeng $\mathrm{Tao}^{1}{ }^{1}$, Xin Chen ${ }^{1}$ \\ 1. Wireless Technology Innovation (WTI) Institute, Key Laboratory of Universal Wireless Communications, Ministry of \\ Education, Beijing University of Posts and Telecommunications (BUPT), Beijing, P.R. China, 100876 \\ 2. University of Edinburgh School of Engineering, Edinburgh EH9 3JL, UK. \\ Email: buptlishiyuan@gmail.com, cuiqimei@bupt.edu.cn,h.haas@ed.ac.uk, taoxf@bupt.edu.cn, \\ chenjiu1986315@gmail.com
}

\begin{abstract}
It is known that Coordinated Multiple Point (CoMP) transmission can increase data rates as more than one copies of signals are coordinated transmitted by multiple points. For the wideband communication system with frequency selective fading channel, the optimal power allocation will be different for each fading block. The Water-Filling (WF) power allocation is optimal for single transmission point. However, it is hard to get closedform optimal solution for the CoMP transmission as the power allocation for fading blocks of one point is simultaneously affected by other coordinated points. This paper analyzes the power allocation of coherent coordinated transmission in CoMP block fading communication system with two transmission points, and the optimal solution is given by Lagrange method. The numerical simulations show that it has a near constant performance gap between the optimal allocation and equal power allocation, and the performance of separate WF power allocation is between optimal allocation and equal power allocation.
\end{abstract}

Keywords- power allocation; Coordinated Multiple Point; CoMP transmission;

\section{INTRODUCTION}

Transmit power allocation among fading blocks is an efficient way to enhance the throughputs of frequency selective wideband communication system. For the power constraint system, the frequency WF (Water-Filling) power allocation is known as the optimal power allocation method for block fading channel [1]. In WF, the fading blocks with large channel gain will be allocated more power than blocks with small channel gain. In high SNR (Signal-to-Noise Ratio) region, the capacity of EPA (Equal Power Allocation) is asymptotically to the capacity of WF [2][3]. That means the performance gain achieved by power allocation among frequency-selective subchannels would be disappearing with SNR increasing.

Recently, cooperative MIMO (Multi-Input Multi-Output) is widely discussed for cellular mobile systems [4]. There are mainly two types of cooperative MIMO schemes: fixed/mobile relay [5][6] and CoMP (Coordinated Multiple Point)[7]. The CoMP technique has been introduced into 3GPP LTE-A $\left(3^{\text {rd }}\right.$ Generation Partnership Project Long Term Evolution Advanced) [8] and it is still being discussed in Release 11 SI (Study Item) [9]. In CoMP scheme, multiple BSs (Base Station), sharing channel and data information between each other, coordinated transmit signals to one or multiple UEs (User Equipment).

Many transmission and reception strategies are researched for CoMP scheme [10-12]. Most of former researches are focus precoding design on flat channel or resource allocation in multiple BSs and UEs. However, while CoMP transmission is adopted in the frequency selective fading channels, power allocation between fading blocks also need to be paid attention because simple power allocation strategies can not touch the maximum throughputs brought by optimal power allocation. Moreover, unlike the case of single BS transmission, the power allocation among fading blocks for CoMP is simultaneously related to both multiple BSs and multiple fading blocks. For the scheme of non-coherent downlink coordinated transmission with single transmitted antenna, Reference [13-15] gives the exact solution form of optimal power allocation among fading blocks.

In this paper, the scheme of coherent downlink coordinated transmission with single transmitted antenna is studied. The optimal power allocation for objective of maximum throughputs is got by Lagrange method. Further more a fast search method is proposed for the solution to reduce computing complexity.

The paper is organized as follows. Section II introduces CoMP block fading communication system with coherent coordinated transmission. Section III gives the optimal power allocation and proposes a fast search method. Section IV gives the numerical simulation. Section V provides some conclusions.

\section{SYSTEM MODEL}

In this paper, the CoMP transmission with frequency selective fading channel is considered. It assumed a certain user is served by two BSs as depicted in Figure 1. In this model, data and control information are shared between 2 BSs through wired connection. The transmission occupy a frequency selective channel of bandwidth $B$, and the whole band can divide into $n$ sub-channels of bandwidth $\Delta B$, where channel response is constant over each sub-channel, so the frequency downlink channel can be expressed as 


$$
H_{i}(f)=\left[h_{i 1} e^{j \phi_{i 1}}, \cdots, h_{i n} e^{j \phi_{i n}}\right] \quad(i=1,2),
$$

where subscript $i$ is the sequence number of two BSs, $h_{i k}$ is the amplitude response of $k$-th sub-channel, and $\phi_{i k}$ is phase shifting of $k$-th sub-channel.
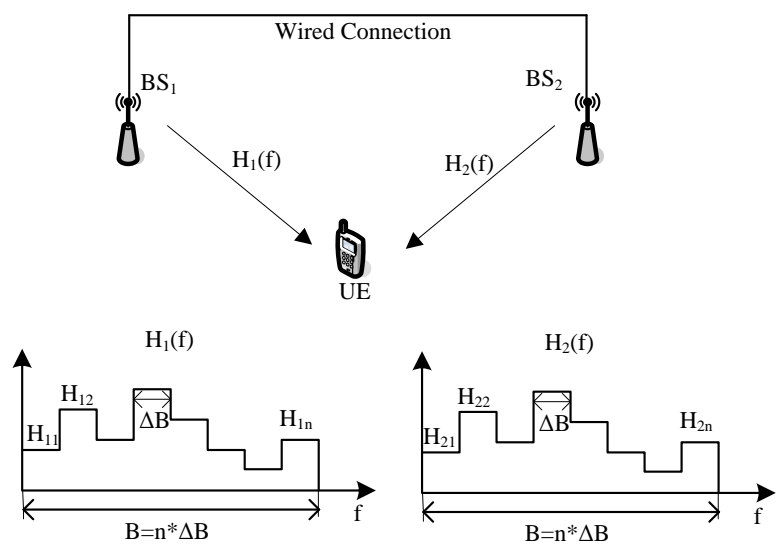

Figure 1. CoMP Block Fading Transmission Scheme

It is assumed that BS knows the perfect downlink channel. So BS can adjust the phase of transmission signals and then the coordinated coherent transmission is performed, which means the signals of two transmit point are adjusted to same phase while UE receive these signals.

So the transmit signal of $k$-th sub-channel is

$$
x_{i k}=p_{i k} e^{-j \phi_{i k}} s_{k}(i=1,2),
$$

where $p_{i k}$ is the signal amplitude of $k$-th sub-channel, $\phi_{i k}$ is phase adjusting factor of $k$-th sub-channel, $s_{k}$ is normalized transmit symbol of $k$-th sub-channel and the two BSs send same symbol in same sub-channel. The maximum power is constraint in the whole transmission band for each BS,

$$
\sum_{k=1}^{n} p_{i k}^{2} \leq P_{i},
$$

where $P_{i}$ is the maximum transmit power of $i$-th BS.

The received coherent coordinated signal of UE is

$$
y_{k}=\left(p_{1 k} h_{1 k}+p_{2 k} h_{2 k}\right) s_{k}+n_{k},
$$

where $n_{k}$ is the system noise with variance $\sigma_{k}^{2}$ of $k$-th subchannel.

So the total throughput of UE is

$$
C=\sum_{k=1}^{n} \log _{2}\left(1+\left(p_{1 k} h_{1 k}+p_{2 k} h_{2 k}\right)^{2} / \sigma_{k}^{2}\right)
$$

\section{OPTIMIZATION SOLUTION}

To maximum the throughput, the power for each subchannels need to be carefully allocated as the channel response of each sub-channel is different. So the problem can be rewrite as optimization problem.

$$
\begin{array}{ll}
\max & C=\sum_{k=1}^{n} \log _{2}\left(1+\left(p_{1 k} h_{1 k}+p_{2 k} h_{2 k}\right)^{2} / \sigma_{k}^{2}\right) \\
\text { s.t. } & \sum_{k=1}^{n} p_{1 k 1}^{2} \leq P_{1} ; \\
& \quad \sum_{k=1}^{n} p_{2 k}^{2} \leq P_{2} ;
\end{array}
$$

The Lagrange function is adopted here to solve this optimization problem,

$$
\begin{aligned}
& L\left(p_{i k}, \lambda_{1}, \lambda_{2}\right)= \\
& \sum_{k=1}^{n} \log _{2}\left(1+\left(p_{1 k} h_{1 k}+p_{2 k} h_{2 k}\right)^{2} / \sigma_{k}^{2}\right)-\lambda_{1} \sum_{k=1}^{n} p_{1 k 1}^{2}-\lambda_{2} \sum_{k=1}^{n} p_{2 k}^{2}
\end{aligned}
$$

The local optimal solution can be got while the first-order derivative of Lagrange function equals to zero. Let $L\left(p_{i k}, \lambda_{1}, \lambda_{2}\right)=0$, it can get a series of equations (8) and (9),

$$
\begin{aligned}
& \frac{2 h_{1 k}\left(p_{1 k}+p_{2 k} h_{2 k}\right) / \sigma_{k}^{2}}{1+\left(p_{1 k} h_{1 k}+p_{2 k} h_{2 k}\right)^{2} / \sigma_{k}^{2}}+2 \lambda_{1} p_{1 k}=0 ; \quad(k=1, \ldots, n) \\
& \frac{2 h_{2 k}\left(p_{1 k} h_{1 k}+p_{2 k} h_{2 k}\right) / \sigma_{k}^{2}}{1+\left(p_{1 k} h_{1 k}+p_{2 k} h_{2 k}\right)^{2} / \sigma_{k}^{2}}+2 \lambda_{2} p_{2 k}=0 ; \quad(k=1, \ldots, n)
\end{aligned}
$$

It is easy to know the maximum throughputs will be got while all the power of BS is allocated, so the optimal solution is at the upper boundary of constraint and the constraint can be rewrite as

$$
\sum_{k=1}^{n} p_{1 k}^{2}=P_{1} ; \sum_{k=1}^{n} p_{2 k}^{2}=P_{2}
$$

From equation (8) and (9), the relevance of allocated powers of two BSs in a sub-channel can be got

$$
\frac{p_{1 k}}{p_{2 k}}=\frac{\lambda_{2} h_{1 k}}{\lambda_{1} h_{2 k}}(k=1, \ldots, n) .
$$

Define $\lambda=\lambda_{1} / \lambda_{2}$, then substitute (11) into (8), it can get

$$
p_{1 k}^{2}=\frac{-\frac{\left(h_{1 k}^{2}+\lambda h_{2 k}^{2}\right)}{\lambda_{1}}-\sigma_{k}^{2}}{\left(h_{1 k}^{2}+\lambda h_{2 k}^{2}\right)^{2} / h_{1 k}^{2}}(k=1, \ldots, n)
$$

Then substitute it into constraint (10), it can get

$$
\lambda_{1}=-\frac{\sum_{k=1}^{n} \frac{h_{1 k}^{2}}{\left(h_{1 k}^{2}+\lambda h_{2 k}^{2}\right)}}{P_{1}+\sum_{k=1}^{n} \frac{\sigma_{k}^{2}}{\left(h_{1 k}^{2}+\lambda h_{2 k}^{2}\right)^{2} / h_{1 k}^{2}}}
$$

Similarly to $p_{2 k}^{2}$ and $\lambda_{2}$, it can get equations (14) and (15) 


$$
\begin{gathered}
p_{2 k}^{2}=\frac{-\frac{\left(h_{1 k}^{2} / \lambda+h_{2 k}^{2}\right)}{\lambda_{2}}-\sigma_{k}^{2}}{\left(h_{1 k}^{2} / \lambda+h_{2 k}^{2}\right)^{2} / h_{2 k}^{2}}(k=1, \ldots, n) \\
\lambda_{2}=-\frac{\sum_{k=1}^{n} \frac{h_{2 k}^{2}}{\left(h_{1 k}^{2} / \lambda+h_{2 k}^{2}\right)}}{P_{2}+\sum_{k=1}^{n} \frac{\sigma_{k}^{2}}{\left(h_{1 k}^{2} / \lambda+h_{2 k}^{2}\right)^{2} / h_{2 k}^{2}}}
\end{gathered}
$$

Solve the two equations (13) and (15), $\lambda_{1}$ and $\lambda_{2}$ can be got.

Due to the complexity of equations (13) and (15), it is difficult to got the closed-form solution for $\lambda_{1}$ and $\lambda_{2}$. The value of them can be got by two-dimensional search. To reduce the computing complexity of two-dimensional search, a onedimensional search method for $\lambda$ is proposed, then $\lambda_{1}, \lambda_{2}$ can be got through equation (13) and (15).

The value of $\lambda$ can be got as follows. Equation (13) divide equation (15), it can get

$$
\frac{\sum_{k=1}^{n} \frac{h_{1 k}^{2}}{\left(h_{1 k}^{2}+\lambda h_{2 k}^{2}\right)}}{\sum_{k=1}^{n} \frac{h_{2 k}^{2}}{\left(h_{1 k}^{2} / \lambda+h_{2 k}^{2}\right)}} * \frac{P_{2}+\sum_{k=1}^{n} \frac{\sigma_{k}^{2}}{\left(h_{1 k}^{2} / \lambda+h_{2 k}^{2}\right)^{2} / h_{2 k}^{2}}}{P_{1}+\sum_{k=1}^{n} \frac{\sigma_{k}^{2}}{\left(h_{1 k}^{2}+\lambda h_{2 k}^{2}\right)^{2} / h_{1 k}^{2}}}=\lambda
$$

Define a function

$$
\begin{aligned}
& f(\lambda)= \\
& \frac{\sum_{k=1}^{n} \frac{h_{1 k}^{2}}{\left(h_{1 k}^{2}+\lambda h_{2 k}^{2}\right)}}{\sum_{k=1}^{n} \frac{h_{2 k}^{2}}{\left(h_{1 k}^{2} / \lambda+h_{2 k}^{2}\right)}} * \frac{P_{2}+\sum_{k=1}^{n} \frac{\sigma_{k}^{2}}{\left(h_{1 k}^{2} / \lambda+h_{2 k}^{2}\right)^{2} / h_{2 k}^{2}}}{P_{1}+\sum_{k=1}^{n} \frac{\sigma_{k}^{2}}{\left(h_{1 k}^{2}+\lambda h_{2 k}^{2}\right)^{2} / h_{1 k}^{2}}}-\lambda
\end{aligned}
$$

While $f(\lambda)=0$, the value of $\lambda$ is the solution of equation (16). The function $f(\lambda)$ has the following property. The proven see appendix.

(1) $f(0)>0$;

(2) $f(+\infty)<0$;

The function $f(\lambda)$ is continuous, so through the property, it easy to know it has at least one solution of $f(\lambda)=0$ in region $(0,+\infty)$. Base on this conclusion, the fast search algorithm based on dichotomy method can be used. The detail of the fast search algorithm for $\lambda$ is described in Algorithm 1 .

Algorithm 1 Fast search algorithm for $\lambda$

1: Initialization: search step $L$; lower bound $\mathrm{S}_{\text {start }}=0$; upper bound $\mathrm{S}_{\text {end }}=L$; error accuracy $\sigma=10^{-6}$;
2: If $f\left(\mathrm{~S}_{\text {end }}+L\right)>0, \mathrm{~S}_{\text {end }}=\mathrm{S}_{\text {end }}+L ; L=10 L$, go to step 2, else go to step 3;

3: If $\operatorname{abs}\left(f\left(\left(\mathrm{~S}_{\text {start }}+\mathrm{S}_{\text {end }}\right) / 2\right)\right)<\sigma$, go to step 5, else go to step 4;

4: If $f\left(\left(\mathrm{~S}_{\text {start }}+\mathrm{S}_{\text {end }}\right) / 2\right)<0, \mathrm{~S}_{\text {end }}=\left(\mathrm{S}_{\text {start }}+\mathrm{S}_{\text {end }}\right) / 2$, else $\mathrm{S}_{\text {start }}=\left(\mathrm{S}_{\text {start }}+\mathrm{S}_{\text {end }}\right) / 2$. Then go to step 3;

5: $\lambda=\left(\mathrm{S}_{\text {start }}+\mathrm{S}_{\text {end }}\right) / 2$, end.

\section{Simulation}

In this section, the numerical simulations are performed to shows the performance of the optimal power allocation for coherent downlink coordinated transmission. In the simulation, system noise $\sigma_{k}^{2}$ is set to constant for all sub-channels. The channel response of each sub-channel is independent and follows gauss distribution. The maximum transmit power of BS is shown through SNR, which equals to $P_{i} / \sum_{k=1}^{n} \sigma_{k}^{2}$, and the same maximum power is set to 2 BSs. The spectrum efficiency shows the performance of power allocation as it removes the influence of bandwidth.

At first, to verify the optimal solution and simulation platform, the comparison between optimal solution and exhaust search of 2 and 3 sub-channels is performed. The simulation results are showed in figure 2 . In the exhaust search, the search step of power allocation for each sub-channel is $0.1 \mathrm{w}$. It can be seen that the optimal solution is accordance to exhaust search, so the solution and simulation platform are right.

To show the performance of optimal power allocation for coherent downlink coordinated transmission, the other two simple power allocation methods are compared with the optimal solution. A) Equal Power Allocation (EPA): the power is equally allocated to each sub-channel for both two BSs; B) Single Water-Filling allocation (SWF): each BS adopts WaterFilling method to allocate the power based on its own channel response respectively.

Figure 3 shows the performance comparison in the normal $\mathrm{SNR}$ region. It can be seen that, in this region, the optimal solution has the best performance and EPA has the worst performance. The performance gap between optimal and EPA is nearly constant, about $0.3 \mathrm{bits} / \mathrm{Hz}$. The performance of SWF is between optimal and EPA. While the SNR is low, the performance of SWF is closed to optimal, and while SNR is high, the performance of SWF is closed to EPA.

Figure 4 shows the performance comparison in very low SNR region. It can be seen that, in this region, the EPA also has worst performance and the performance of SWF is almost same to the optimal. So for low SNR, the SWF method can be adopted to reduce the computing complexity. 


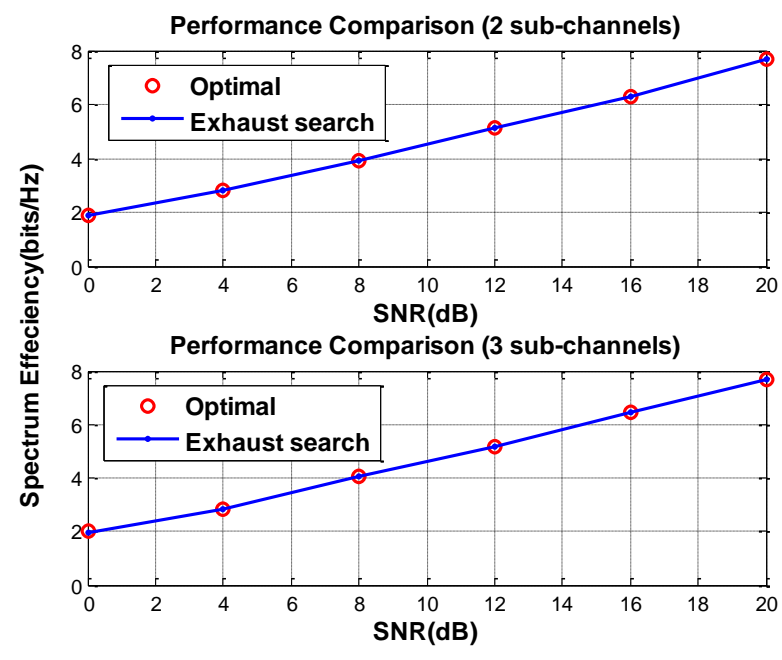

Figure 2. Performance Comparison

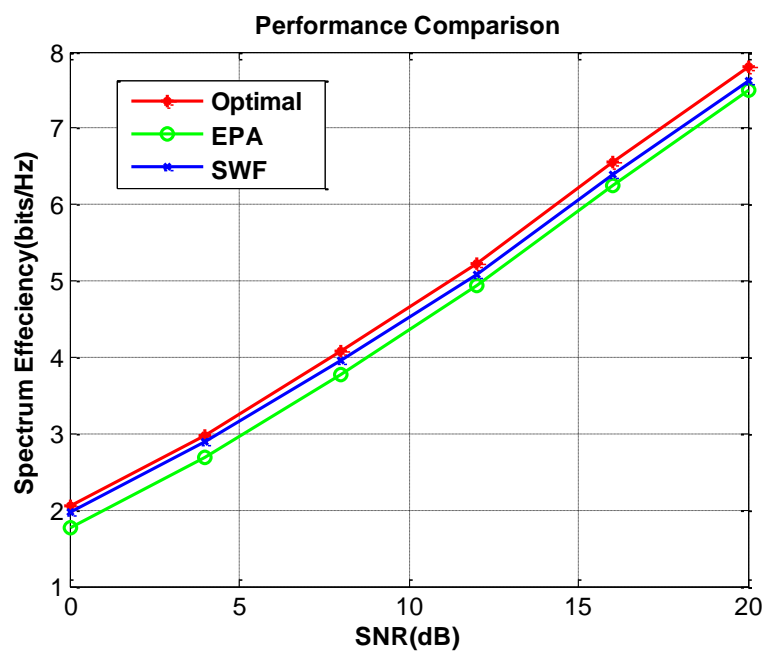

Figure 3. Performance Comparison (normal SNR region)

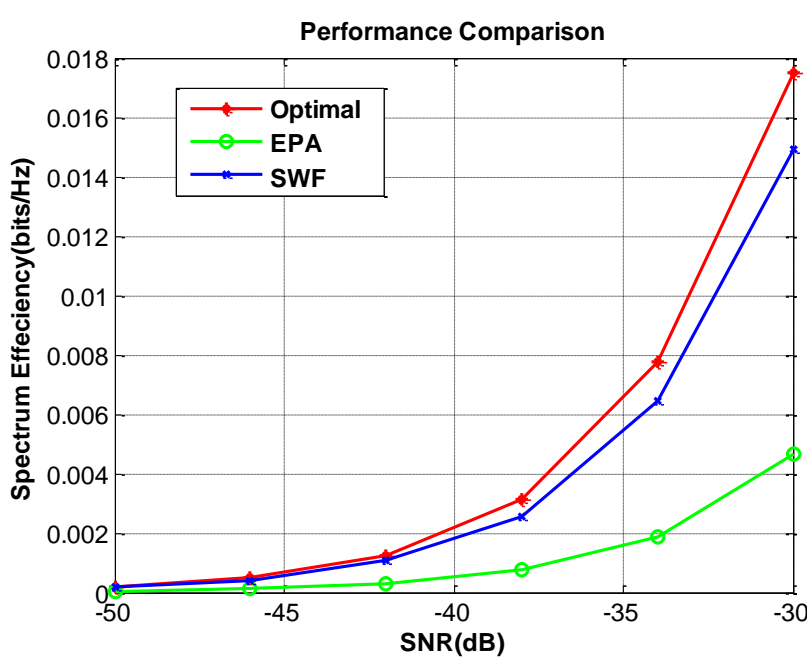

Figure 4. Performance Comparison (low SNR region)

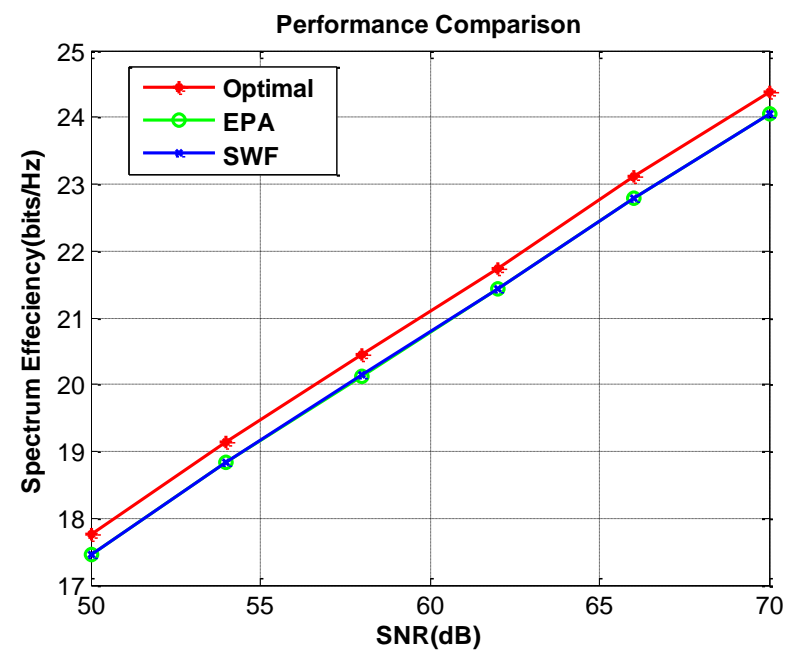

Figure 5. Performance Comparison (high SNR region)

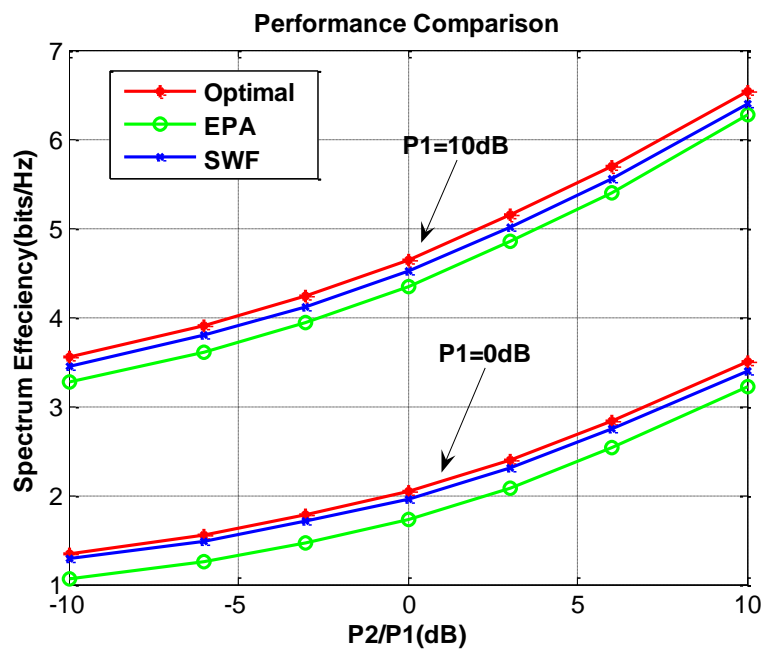

Figure 6. Performance Comparison for different max power constraint

Figure 5 shows the performance comparison in very high SNR region. It can be seen that, in this region, the EPA and SWF have the same performance and the performance gap between optimal and EPA is also constant, about 0.3 bits/Hz.

Figure 6 shows the performance comparison while two BSs have the different max power constraint. The $X$ axis shows the maximum power ratio of two $\mathrm{BSs}, \mathrm{P} 2 / \mathrm{P} 1$ in $\mathrm{dB}$ form. It can be seen that, the EPA also has worst performance, and the performance gap is also about $0.3 \mathrm{bits} / \mathrm{Hz}$ for different power ratio. While $\mathrm{P} 1$ and $\mathrm{P} 2$ are both small, the performance of WPF is much closed to optimal.

\section{CONCLUSION}

In this paper, it analyzes the power allocation for coherent coordinated transmission in block fading communication system of two transmission points, and the optimal solution with fast search method is given. The numerical simulations show that the EPA method has the worst performance, and the performance gap between optimal and EPA is constant, about $0.3 \mathrm{bits} / \mathrm{Hz}$. The performance of SWF method is between 
optimal and EPA. It is closed to optimal in low SNR region and is closed to EPA in high SNR region.

\section{ACKNOWLEDGMENT}

This work is sponsored by the International Scientific and Technological Cooperation Program (Grant No. 2010DFA11060), China-EU International Scientific and Technological Cooperation Program (0902), the National S\&T Major Program (Grant No. 2012ZX03001039), Beijing Municipal Science and Technology Project under Grand D121100002112002, and the Research Councils U.K. (RCUK) under the UK-China Science Bridges Project: R\&D on (B) $4 G$ Wireless Mobile Communications (EP/G042713/1).

\section{APPENDIX}

\section{PROOF THE PROPERTY OF FUNCTION (17)}

The proof will adopt following axiom:

$$
\begin{aligned}
& 1 / 0=\infty ; \\
& 1 / \infty=0 ; \\
& \text { - } \quad \infty^{*} \infty=\infty ; \\
& 1+\infty=\infty ;
\end{aligned}
$$

(1) $f(0)>0$

Substitute $\lambda=0$ into equation (17), then the $f(0)$ equal to

$$
\begin{aligned}
& \frac{\sum_{k=1}^{n} \frac{h_{1 k}^{2}}{\left(h_{1 k}^{2}+0^{*} h_{2 k}^{2}\right)}}{h_{k=1}^{2}} * \frac{P_{2}+\sum_{k=1}^{n} \frac{\sigma_{k}^{2}}{\left(h_{1 k}^{2} / 0+h_{2 k}^{2}\right)^{2} / h_{2 k}^{2}}}{\left.h_{k}^{2} / h_{2 k}^{2}\right)}-0 \\
& P_{1}+\sum_{k=1}^{n} \frac{\sigma_{k}^{2}}{\left(h_{1 k}^{2}+0^{*} h_{2 k}^{2}\right)^{2} / h_{1 k}^{2}} \\
& =\frac{\sum_{k=1}^{n} 1}{0} * \frac{P_{2}}{P_{1}+\sum_{k=1}^{n} \frac{\sigma_{k}^{2}}{h_{1 k}^{2}}} \\
& =+\infty>0
\end{aligned}
$$

The property (1) is proved.

(2) $f(+\infty)<0$

Substitute $\lambda=+\infty$ into equation (17), then the $f(\infty)$ equal to

$$
\begin{aligned}
& \frac{\sum_{k=1}^{n} \frac{h_{1 k}^{2}}{\left(h_{1 k}^{2}+\infty^{*} h_{2 k}^{2}\right)}}{\sum_{k=1}^{n} \frac{h_{2 k}^{2}}{\left(h_{1 k}^{2} / \infty+h_{2 k}^{2}\right)}} * \frac{P_{2}+\sum_{k=1}^{n} \frac{\sigma_{k}^{2}}{\left(h_{1 k}^{2} / \infty+h_{2 k}^{2}\right)^{2} / h_{2 k}^{2}}}{P_{1}+\sum_{k=1}^{n} \frac{\sigma_{k}^{2}}{\left(h_{1 k}^{2}+\infty * h_{2 k}^{2}\right)^{2} / h_{1 k}^{2}}}-\infty \\
& =\frac{0}{\sum_{k=1}^{n} 1} * \frac{P_{2}+\sum_{k=1}^{n} \frac{\sigma_{k}^{2}}{h_{2 k}^{2}}}{P_{1}}-\infty \\
& =-\infty<0
\end{aligned}
$$

The property (2) is proved.

\section{REFERENCES}

[1] Andrea Goldsmith, Wireless Communications,Cambridge University Press, 2005

[2] P. Viswanath, D. N. C. Tse, and V. Anantharam, "Asymptotically optimal water-filling in vector multiple-access channels," IEEE Trans. Inform. Theory, vol. 47, no. 1, pp. 241-267, Jan. 2001

[3] J. Jang and K. B. Lee, "Transmit power adaptation for multiuser OFDM system," IEEE J. Select. Areas Commun., vol. 21, no. 2, pp. 171178,Feb. 2003

[4] Cheng-Xiang Wang; Xuemin Hong; Xiaohu Ge; Xiang Cheng; Gong Zhang; Thompson, J.; , "Cooperative MIMO channel models: A survey," Communications Magazine, IEEE, vol.48, no.2, pp.80-87, February 2010

[5] Shichuan Ma; Yaoqing Yang; Sharif, H.; , "Distributed MIMO technologies in cooperative wireless networks," Communications Magazine, IEEE , vol.49, no.5, pp.78-82, May 2011

[6] D. Soldani and S. Dixit, "Wireless Relays for Broadband Access," IEEE Commun. Mag., vol. 46, no. 3, Mar.2008, pp. 58-66.

[7] M. K. Karakayali, G. J. Foschini, and R. A. Valenzuela, "Network Coordination for Spectrally Efficient Communications in Cellular Systems," IEEE Commun. Mag., vol. 44, no. 8, Aug. 2006, pp. 56-61.

[8] 3GPP, TR36.814(V9.0.0), Further Advancements for E-UTRA Physical Layer Aspects. March 2010.

[9] RP-101425, Revised SID Proposal: Coordinated Multi-Point Operation for LTE, Samsung, 3GPP TSG RAN\#50, December, 2010

[10] Sawahashi, M.; Kishiyama, Y.; Morimoto, A.; Nishikawa, D.; Tanno, M.; , "Coordinated multipoint transmission/reception techniques for LTE-advanced [Coordinated and Distributed MIMO]," Wireless Communications, IEEE, vol.17, no.3, pp.26-34, June 2010

[11] Hui, Dennis; , "Distributed Precoding with Local Power Negotiation for Coordinated Multi-Point Transmission," Vehicular Technology Conference (VTC Spring), 2011 IEEE 73rd, vol., no., pp.1-5, 15-18 May 2011

[12] R1-091493, Coherent and Non-Coherent DL CoMP Transmission for LTE-A, Fujitsu , 3GPP TSG-RAN1 \#56-BIS, March, 2009

[13] B. Luo, Q. Cui, H. Wang and X. Tao, "Optimal joint water-filling for OFDM systems with multiple cooperative power sources," in Proc.of IEEE Globecom' 2010

[14] Qimei Cui, Xueqing Huang, Bing Luo, Xiaofeng Tao, Jun Jiang: Capacity analysis and optimal power allocation for coordinated transmission in MIMO-OFDM systems. SCIENCE CHINA Information Sciences 55(6): 1372-1387, 2012

[15] Qimei Cui, Bing Luo, Xueqing Huang, Alexis Alfredo Dowhuszko, Jun Jiang: Closed-form solution for minimizing power consumption in coordinated transmissions. EURASIP J. Wireless Comm. and Networking 2012: 122, 2012 